\section{Alcohol consumption and suicide}

\section{among middle-aged men in Japan}

\author{
TATSUO AKECHI, MOTOKI IWASAKI, YOSUKE UCHITOMI \\ and SHOICHIRO TSUGANE on behalf of the JPHC Study Group
}

(Cohort I) and 1993-4 (Cohort II), mostly between February and March, to determine socio-demographic characteristics, personal medical history, smoking status, alcohol intake, alcohol-related flushing, physical activity, and other lifestyle-related factors (e.g. food intake). The questionnaire used for Cohort II was modified to include questions on social support.

\begin{abstract}
Background Few studies have investigated large population-based cohorts for an association between alcohol consumption and suicide.
\end{abstract}

Aims To investigate the possible association between alcohol intake and suicide.

\section{Method We used nationwide population-based cohort data from the Japan Public Health Centre-Based Prospective Study cohort. A total of 43383 men aged 40-69 years participated. Death from suicide was defined according to ICD-10 as codes $\times 60-\times 84$}

Results During the 367817 personyears of follow-up, there were 168 suicides. Compared with occasional drinkers, the pooled multivariate relative risk ( $\mathrm{RR}$; with $95 \% \mathrm{Cl}$ ) of suicide for nondrinkers and regular heavy drinkers was 2.3 (I.2-4.6) and 2.3 (I.2-4.7) respectively. The RR increased with the amount of alcohol consumed ( $P$ for trend $=0.004$ ).

\section{Conclusions There is a U-shaped} association between alcohol consumption and subsequent suicide.

Declaration of interest None. Funding detailed in Acknowledgements.
It was recently reported that more than 30000 people in Japan die by suicide annually (the suicide rate in 1998 was 25.4 per 100000 ), one of the highest suicide rates in the world, and that more than $40 \%$ of Japanese suicide victims are middle-aged (in their 40s and 50s; Takahashi, 2002). Heavy alcohol consumption is among the well-known risk factors for suicide (Harris \& Barraclough, 1997; Mann, 2002), and the lifetime suicide rate is estimated to be $7 \%$ among those who misuse alcohol (Inskip et al, 1998). However, few studies have investigated the association between alcohol consumption and subsequent completion of suicide using a validated measurement of alcohol consumption in a large population-based cohort. The purpose of the present study was to investigate the association between alcohol consumption and suicide based on the data obtained from middle-aged males in the Japan Public Health Centre-Based Prospective Study (JPHC Study) cohort.

\section{METHOD}

\section{Study cohort}

This study was conducted as part of the JPHC study, which began in 1990 for Cohort I and in 1993 for Cohort II. The study population was defined as all residents aged 40-59 years in Cohort I and 40-69 years in Cohort II at baseline who had registered their addresses in 27 municipalities supervised by nine public health centres. We established a population-based cohort of 57714 men (27063 in Cohort I and 30651 in Cohort II). Details of the study design have been reported elsewhere (Watanabe et al, 2001). The JPHC study was approved by the institutional review board of the National Cancer Centre, Tokyo, Japan.

\section{Baseline questionnaire survey}

A self-administered questionnaire was distributed to all cohort members in 1990

\section{Alcohol consumption}

In Cohort I, we defined non-drinkers $(<1$ day/month), occasional drinkers (1-3 days/month) and regular drinkers (1-2 days/week or more) based on the frequency of alcohol consumption. Subjects in Cohort II were asked about their alcohol consumption habits, i.e. whether they were never, ex-, or current drinkers. To match the variables of alcohol intake in the two cohorts, the 'non-drinker' category was defined as never drinkers and ex-drinkers combined in Cohort II. Current drinkers were divided into occasional drinkers (13 days/month) and regular drinkers (1-2 days/week or more), based on the frequency of consumption. Among regular drinkers, weekly ethanol intake was calculated by multiplying frequency per week by the amount of alcohol consumed daily. The amount of ethanol in each alcoholic drink was calculated as follows: $180 \mathrm{ml}$ sake (rice wine) as $23 \mathrm{~g}$ ethanol, $180 \mathrm{ml}$ shochu or awamori (white spirits) as $36 \mathrm{~g}$, $633 \mathrm{ml}$ beer as $23 \mathrm{~g}, 30 \mathrm{ml}$ whisky or bran$\mathrm{dy}$ as $10 \mathrm{~g}$ and $60 \mathrm{ml}$ wine as $6 \mathrm{~g}$. The method for calculating ethanol intake has been reported in detail elsewhere (Tsugane et al, 1999; Sobue et al, 2001). Participants were classified into six groups: nondrinkers, occasional drinkers, and four groups of regular drinkers according to the quartile of weekly ethanol intake (<138 g/week, 138-251 g/week, 252$413 \mathrm{~g} /$ week and $\geqslant 414 \mathrm{~g} /$ week).

To evaluate the validity of the estimation of alcohol intake from the baseline questionnaires, we collected four 7-day dietary records (a total of 28 days) from volunteers in each cohort. Spearman's rank correlations for estimated alcohol intake between the baseline questionnaires and dietary records were 0.79 in 94 men in Cohort I (Tsubono et al, 2003) and 0.59 in 176 men in Cohort II (unpublished data). This indicates that our questionnaire had moderate validity for ranking participants according to their alcohol consumption. 


\section{Facial flushing}

Previous studies have indicated that approximately half of those from East Asia have very low aldehyde dehydrogenase 2 (ALDH2) activity. This enzyme limits alcohol intake by means of the unpleasant symptoms that occur after alcohol consumption owing to the low capacity to metabolise aldehyde (Ferguson \& Goldberg, 1997). Facial flushing after alcohol consumption is an excellent indicator of the presence of inactive ALDH2 (Takeshita et al, 1993; Yokoyama et al, 1997). Moreover, facial flushing is well known to be associated with reduction in both drink ing and progression to alcoholism (Higuchi et al, 1992). Thus since the mechanism underlying the association of alcohol consumption and suicide is not clear (Hufford, 2001) we also investigated the relationship between alcohol consumption and suicide in individuals with and without facial flushing. A facial flushing questionnaire was used, which Yokoyama et al (1997) reported had good reliability (high sensitivity and specificity) for inactive ALDH2.

\section{Follow-up and identification of suicide}

We followed all participants from baseline until 31 December 1999 for Cohort I and 31 December 2000 for Cohort II. Data on migration were obtained from the residential registry annually. Of the initial participants, 1966 (4.3\%) moved out of the study area and $36(0.08 \%)$ were lost to follow-up. Data on deaths of participants who remained in their original area were based on death certificates from the local public health centre. For those who died after moving from their original area, the cause of death was extracted from death certificates kept by the Ministry of Health, Labour and Welfare, Japan. Death by suicide was defined according to ICD10 (World Health Organization, 1992) as codes X60-X84. All death certificates in Japan are submitted to a local government office and forwarded to the public health centre in the area of residence. Mortality data are then sent to the Ministry of Health, Labour and Welfare and coded for National Vital Statistics. Registration of deaths is mandatory in Japan under the Family Registration Law and death certificates must be completed by a licensed physician. The verdict of suicide is based on the results of the medico-legal examination by a licensed physician and the police investigation as required by Japanese law. Thus, the data are believed to be sufficient in terms of quality and completeness.

\section{Statistical analysis}

Person-years of follow-up were calculated from baseline until the date of suicide, the most recent date when survival status was confirmed in censored cases, and at the end of the study periods (31 December 1999 for Cohort I and 31 December 2000 for Cohort II) for survivors, whichever occurred first. The crude suicide rate was calculated by dividing the number of suicide cases by the number of person-years. For each cohort, the Cox proportional hazards model was used to estimate relative risk (RR) and 95\% confidence intervals (CIs) of suicide related to alcohol consumption at baseline using the SAS program version 8.2 for Windows (PROC PHREG; SAS Institute, Cary, North Carolina, USA). The RRs were adjusted for age, area, living alone and unemployment. Linear trends were tested in the Cox proportional hazards models by treating the categories as ordinal variables. The interaction test was performed for facial flushing status. All $P$ values reported are two-sided and the significance level was set at $P<0.05$.

We used the fixed-effects model to combine the $\log _{\mathrm{e}} \mathrm{RRs}$; the cohort-specific RRs were weighted by the inverse of their variance after testing for heterogeneity among cohorts (Petitti, 1994). The pooled $P$ value for the interaction test was calculated by the sum of logs method (Becker, 1994).

\section{RESULTS}

A total of 45452 men (79\%), 20658 in Cohort I and 24794 in Cohort II, returned the questionnaire. We excluded those for whom information on alcohol consumption was incomplete (2069 men $(4.6 \%)$, including 6 who died by suicide). Among 367817 person-years of observation of 43383 men (mean follow-up $=8.5$ years), there were $168(0.39 \%)$ suicides (97 in Cohort I and 71 in Cohort II). At baseline (Table 1), regular drinkers in Cohorts I and II who consumed more than $414 \mathrm{~g}$ of ethanol per week were more likely to smoke and less likely to have facial flushing than occasional drinkers. Non-drinkers were more likely to be receiving medication and to be unemployed. The proportion of men taking medication was highest among the exdrinkers. Data on social support variables were available for Cohort II. Non-drinkers were less likely to have close friends and confidants than others. These data were not available for Cohort I.

The association between alcohol consumption at baseline and subsequent death by suicide is shown in Table 2. Compared with occasional drinkers, the pooled multivariate RRs for non-drinkers and regular drinkers who consumed more than $414 \mathrm{~g}$ of ethanol per week were $2.3(95 \%$ CI $1.2-4.6)$ and 2.3 (95\% CI 1.2-4.7) respectively. Among non-drinkers in Cohort II, ex-drinkers had a significantly increased risk of suicide compared with occasional drinkers, but never drinkers did not, even after adjusting for potential confounders. Among drinkers the risk of suicide increased with the amount of ethanol consumed, and the trend test revealed statistical significance $(P$ for trend $=0.004)$. There is a U-shaped association between alcohol consumption and subsequent death by suicide (Fig. 1).

To maintain statistical power, we recategorised drinkers into three groups. Because groups of occasional drinkers and regular drinkers who consumed $138-251 \mathrm{~g}$ ethanol showed almost the same risk as groups of regular drinkers who consumed less than $138 \mathrm{~g}$ and $252-413 \mathrm{~g}$ ethanol respectively, we combined these categories. Thereafter, we analysed the association between alcohol consumption and subsequent death by suicide among drinkers further adjusting for flushing status. Regular heavy drinkers who consumed more than $414 \mathrm{~g}$ of ethanol per week had a significantly higher risk of suicide than those who drank occasionally or consumed less than $138 \mathrm{~g}$ of ethanol per week (pooled multivariate $\mathrm{RR}=2.3$, 95\% CI 1.4-3.8).

Among men for whom data on alcoholrelated flushing status were available $(n=39241), 43.2 \%$ reported being nonflushers and $56.8 \%$ being flushers. Flushers did not have a significantly increased risk of suicide compared with non-flushers (pooled multivariate $\mathrm{RR}=1.3,95 \% \mathrm{CI} 0.9-1.8$ ). To determine whether alcohol consumption differed in terms of subsequent death by suicide between non-flushers and flushers, we analysed the data in separate facial flushing strata. Among non-flushers, a non-significant elevated risk was observed for regular drinkers who consumed more than $414 \mathrm{~g}$ of ethanol per week (pooled multivariate $\mathrm{RR}=2.1,95 \%$ CI $0.9-4.7$ ) compared with those who drank occasionally or consumed less than $138 \mathrm{~g}$ of ethanol 
Table I Baseline characteristics of Cohorts I and II of the Japan Public Health Centre-Based Prospective Study Group (men aged 40-69) according to alcohol consumption

\begin{tabular}{|c|c|c|c|c|c|c|c|}
\hline & \multicolumn{2}{|c|}{ Non-drinkers } & \multirow{2}{*}{$\begin{array}{l}\text { Occasional } \\
\text { drinkers }\end{array}$} & \multicolumn{4}{|c|}{ Regular drinkers', weekly ethanol intake (g) } \\
\hline & $\begin{array}{l}\text { Never } \\
\text { drinkers }\end{array}$ & Ex-drinkers & & $<138 g$ & $138-25 \mid g$ & $252-413 \mathrm{~g}$ & $\geqslant 414 \mathrm{~g}$ \\
\hline \multicolumn{8}{|l|}{ Cohort I } \\
\hline Number of men & \multicolumn{2}{|c|}{4287} & 2200 & 2978 & 3785 & 3436 & 3522 \\
\hline Age, years: mean & \multicolumn{2}{|c|}{50.2} & 49.0 & 49.3 & 49.3 & 49.6 & 49.3 \\
\hline Living alone, $\%$ & \multicolumn{2}{|c|}{3.0} & 3.0 & 2.7 & 2.4 & 1.9 & 2.1 \\
\hline Unemployed, \% & \multicolumn{2}{|c|}{8.5} & 3.2 & 3.0 & 1.8 & 2.0 & 2.5 \\
\hline Any medicines, \% & \multicolumn{2}{|c|}{27.4} & 19.0 & 20.9 & 21.3 & 22.9 & 24.6 \\
\hline Current smokers, \% & \multicolumn{2}{|c|}{47.5} & 46.3 & 45.4 & 52.7 & 59.4 & 64.4 \\
\hline Flushers, \% & \multicolumn{2}{|c|}{65.8} & 64.7 & 57.5 & 51.9 & 46.5 & 44.0 \\
\hline \multicolumn{8}{|l|}{ Cohort II } \\
\hline Number of men & 5018 & 1021 & 1966 & 3614 & 3847 & 3605 & 4104 \\
\hline Age, years: mean & 56.5 & 59.2 & 52.2 & 52.8 & 54.1 & 53.4 & 53.6 \\
\hline Living alone, $\%$ & 4.1 & 4.6 & 3.9 & 3.5 & 2.8 & 3.3 & 3.3 \\
\hline Unemployed, \% & 13.8 & 23.7 & 5.3 & 6.1 & 6.3 & 4.3 & 5.1 \\
\hline $\begin{array}{l}\text { Having no person who makes you } \\
\text { feel comfortable and/or safe, } \%\end{array}$ & 26.0 & 25.7 & 24.0 & 18.8 & 22.0 & 22.4 & 24.6 \\
\hline $\begin{array}{l}\text { Having no friend who you talk to } \\
\text { once a week or more, } \%\end{array}$ & 18.3 & 20.7 & 15.4 & 14.8 & 15.8 & 15.3 & 14.9 \\
\hline $\begin{array}{l}\text { Having no person who agrees } \\
\text { with and/or supports your } \\
\text { actions and/or ideas, } \%\end{array}$ & 19.4 & 20.3 & 14.1 & 13.0 & 13.5 & 13.4 & 16.4 \\
\hline $\begin{array}{l}\text { Having no person to whom you } \\
\text { can talk about personal } \\
\text { feelings or secrets, } \%\end{array}$ & 25.0 & 24.3 & 23.3 & 19.8 & 22.5 & 21.5 & 22.9 \\
\hline Any medicines, $\%$ & 35.6 & 65.8 & 26.5 & 28.7 & 30.1 & 28.4 & 29.0 \\
\hline Current smokers, \% & 47.5 & 43.3 & 47.5 & 43.8 & 50.8 & 58.1 & 61.9 \\
\hline Flushers, \% & 70.1 & 48.0 & 62.3 & 53.0 & 43.8 & 36.6 & 32.5 \\
\hline
\end{tabular}

I. Drinking at least I day/week.

per week; a borderline significant positive trend was also found ( $P$ for trend $=0.07$; Fig. 2). Among flushers, regular drinkers who consumed more than $414 \mathrm{~g}$ of ethanol per week had a significantly increased risk (pooled multivariate $\mathrm{RR}=2.5,95 \% \mathrm{CI}$, 1.3-4.8) compared with those who drank occasionally or consumed less than $138 \mathrm{~g}$ of ethanol per week, and a positive trend was observed ( $P$ for trend $=0.007$ ). The interaction test for facial flushing status did not show statistical significance $(P=0.96)$.

To exclude effects of other potential confounders, the RRs were further adjusted for medication, past history of any chronic diseases, self-reported stress, smoking status, body mass index, and sports and physical exercise in leisure time. However, the results did not substantially change after adjustment (data not shown). To eliminate effects of preclinical diseases, we performed analyses that excluded the first 2 years of follow-up. The pooled RR in non-drinkers was attenuated and the statistical significance of the association disappeared (pooled multivariate $\mathrm{RR}=1.8$, 95\% CI 0.9-3.7). In Cohort II, the RRs were attenuated in both never drinkers (multivariate $\mathrm{RR}=1.2$, 95\% CI $0.3-4.4$ ) and ex-drinkers (multivariate $\mathrm{RR}=6.0$, 95\% CI 1.6-22.7) but the statistical significance was still observed in the ex-drinkers. To account for the possible under-reporting of suicide cases, the association of alcohol consumption with suicide was examined including deaths from undetermined causes (ICD-10 code Y10-Y34, $n=19$ ) as suicide cases. However, we observed a similar U-shaped association (data not shown).

\section{DISCUSSION}

\section{Association between alcohol consumption and suicide}

This is the first study to show a U-shaped association between alcohol consumption and subsequent death by suicide on the basis of validated measurement of alcohol consumption in two large Japanese population-based middle-aged male cohorts.

To the best of our knowledge, only five cohort studies have investigated the association between alcohol consumption and subsequent suicide (Paffenbarger et al, 1969; Ross et al, 1990; Andreasson et al, 1991; Klatsky \& Armstrong, 1993; Turvey et al, 2002) but their findings were inconsistent. Three of these reports indicated that individuals consuming large amounts of alcohol were more likely to die by suicide than those who never drank or consumed moderate amounts (Ross et al, 1990; Andreasson et al, 1991; Klatsky \& Armstrong, 1993). The other two studies found no significant association (Paffenbarger et al, 1969; Turvey et al, 2002). Our present findings indicate that heavy drinkers among middle-aged Japanese men, who constitute 
Table 2 Relative risk of suicide according to alcohol consumption

\begin{tabular}{|c|c|c|c|c|c|c|c|c|c|}
\hline & \multirow{2}{*}{$\begin{array}{c}\text { Never } \\
\text { drinkers }\end{array}$} & \multirow{2}{*}{$\begin{array}{c}\text { Ex- } \\
\text { drinkers }\end{array}$} & \multirow{2}{*}{$\begin{array}{c}\text { Non- } \\
\text { drinkers }\end{array}$} & \multirow{2}{*}{$\begin{array}{l}\text { Occasional } \\
\text { drinkers }\end{array}$} & \multicolumn{4}{|c|}{ Regular drinkers', weekly ethanol intake (g) } & \multirow{2}{*}{$\begin{array}{c}P \text { for trend } \\
\text { among } \\
\text { drinkers }\end{array}$} \\
\hline & & & & & $<138 \mathrm{~g}$ & $138-25 \mid \mathrm{g}$ & $252-413 \mathrm{~g}$ & $\geqslant 414 \mathrm{~g}$ & \\
\hline \multicolumn{10}{|l|}{ Cohort I } \\
\hline Number of suicides & & & 29 & 7 & 10 & 14 & 12 & 25 & \\
\hline Person-years & & & 41216 & 21310 & 28956 & 36778 & 33349 & 33864 & \\
\hline Crude suicide rate & & & 70.4 & 32.8 & 34.5 & 38.1 & 36.0 & 73.8 & \\
\hline $\begin{array}{l}\text { Age/area-adjusted RR } \\
(95 \% \mathrm{Cl})^{2}\end{array}$ & & & $2.2(0.9-4.9)$ & 1.0 & I.I (0.4-2.8) & $1.2(0.5-3.0)$ & $1.2(0.5-3.0)$ & $2.3(0.98-5.4)$ & 0.02 \\
\hline Multivariate RR $(95 \% \mathrm{Cl})^{3}$ & & & $2.2(0.96-5.1)$ & 1.0 & $1.0(0.4-2.6)$ & $1.2(0.5-3.1)$ & $1.2(0.5-3.1)$ & $2.3(0.99-5.4)$ & 0.01 \\
\hline \multicolumn{10}{|l|}{ Cohort II } \\
\hline Number of suicides & 13 & 10 & 23 & 3 & 9 & 10 & 10 & 16 & \\
\hline Person-years & 36866 & 7116 & 43982 & 14855 & 27065 & 28744 & 27007 & 30691 & \\
\hline Crude suicide rate & 35.3 & 140.5 & 52.3 & 20.2 & 33.3 & 34.8 & 37.0 & 52.1 & \\
\hline $\begin{array}{l}\text { Age/area-adjusted RR } \\
(95 \% \mathrm{Cl})^{2}\end{array}$ & $1.8(0.5-6.2)$ & $7.0(1.9-25.9)$ & $2.6(0.8-8.7)$ & 1.0 & $1.6(0.4-6.1)$ & $1.7(0.5-6.1)$ & $1.7(0.5-6.4)$ & $2.5(0.7-8.6)$ & 0.13 \\
\hline Multivariate RR $(95 \% \mathrm{Cl})^{3}$ & $1.7(0.5-6.2)$ & $6.7(1.8-25.0)$ & $2.5(0.7-8.5)$ & 1.0 & $1.6(0.4-6.1)$ & $1.7(0.5-6.1)$ & $1.8(0.5-6.5)$ & $2.5(0.7-8.7)$ & 0.12 \\
\hline \multicolumn{10}{|c|}{ Pooled estimates } \\
\hline Multivariate RR $(95 \% \mathrm{Cl})^{3}$ & & & $2.3(1.2-4.6)$ & 1.0 & $1.2(0.5-2.6)$ & $1.4(0.6-2.9)$ & $1.3(0.6-2.9)$ & $2.3(1.2-4.7)$ & 0.004 \\
\hline
\end{tabular}

RR, relative risk.

I. Drinking at least I day/week.

2. Adjusted for age at baseline and area (nine public health centres).

3. Adjusted for age at baseline, area (nine public health centres), living alone (no, yes), and employment status (employed, unemployed).

the majority of Japanese suicide victims, are not the only group vulnerable to subsequent death by suicide. Non-drinkers are also at risk. The increased risk for death by suicide among non-drinkers was an unexpected finding, and there are several possible explanations.

First, because the findings were somewhat attenuated after excluding the first 2 years of follow-up, non-drinkers might have several underlying physical diseases, which could be risk factors for later suicide. Second, one epidemiological study investigating drinking habits and depression among middle-aged and elderly Japanese males revealed a significantly

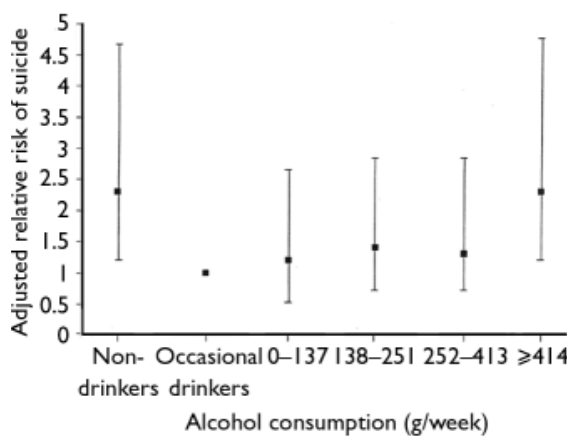

Fig. I Relative risk of suicide after adjustment for age, area, living alone and employment status according to alcohol consumption. Bars represent $95 \%$ confidence intervals. higher degree of depression among nondrinkers than among moderate and even problem drinkers (Sato et al, 1990). Underlying depression in non-drinkers may contribute to the higher risk for suicide. Third, non-drinkers in Cohort II perceived less social support, for example, in the form of close friends and confidants, and this might contribute to the increased risk of subsequent suicide (Heikkinen et al, 1993). There might also be other factors associated with a non-drinking lifestyle, such as specific personality traits, coping strategies, religious beliefs and a past history of alcohol dependence, although we do not have clear empirical data supporting the association. Finally, the difference between never drinkers and ex-drinkers which are both classed as non-drinkers in the present study, should be considered more carefully because each showed different RR compared with occasional drinkers in Cohort II. An increased risk of suicide might be observed only in exdrinkers but not in never drinkers. Our findings, like those of other health outcomes (de Lorimier, 2000), suggest a U-shaped association between alcohol consumption and suicide. However, further research is needed to determine why nondrinkers are more likely to die by suicide than moderate drinkers.

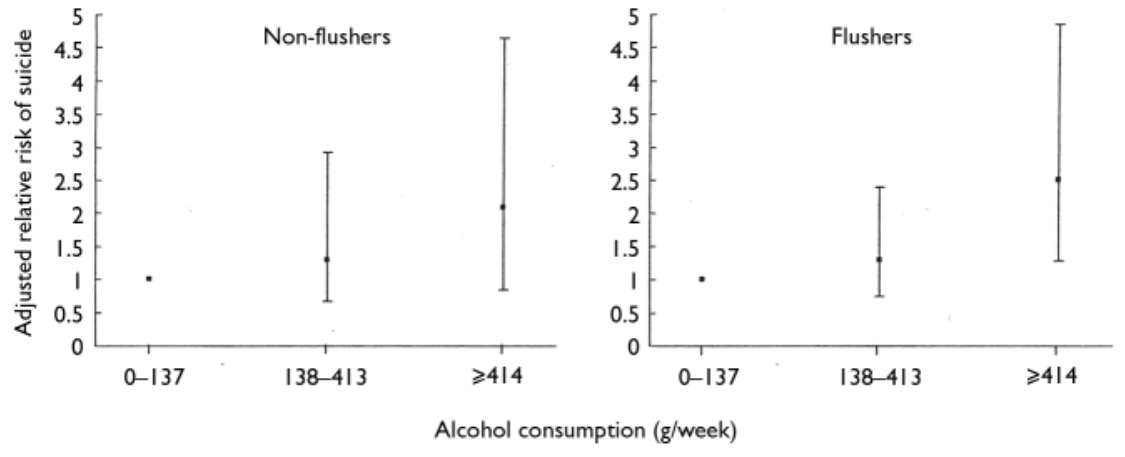

Fig. 2 Relative risk of suicide after adjustment for age, area, living alone and employment status according to alcohol consumption in flushers and non-flushers. Bars represent $95 \%$ confidence intervals. 


\section{Alcohol-related flushing and suicide}

The dose-response effect of alcohol consumption on death by suicide was significant in regular drinkers with alcoholrelated flushing, although a borderline significance was also observed among non-flushers and the interaction test for facial flushing did not yield statistically significant results. Several recent studies have demonstrated potential roles for genetic factors, including polymorphisms, in psychiatric disorders that are associated with suicide behaviours (Hasegawa et al, 2002; Huang et al, 2003) but the current findings do not suggest any clearly different effect of alcohol consumption on suicide among non-flushers (active ALDH2) and flushers (inactive ALDH2).

\section{Methodological advantages of this study}

Our study had several methodological advantages. First, the prospective design made it possible to avoid recall bias. Most epidemiological studies of alcohol consumption and suicide have used a retrospective design with proxy respondents. Second, this study included a large population-based sample with a high response rate (approximately $80 \%$ ) and a very low rate of loss to follow-up. Third, we measured alcohol consumption using a validated questionnaire, whereas most previous cohort studies did not use a validated measurement for alcohol consumption (Paffenbarger et al, 1969; Ross et al, 1990; Andreasson et al, 1991; Turvey et al, 2002). Fourth, the similar findings in the two cohorts strengthens the results.

\section{Limitations}

Our study had some limitations. First, several important items of data were not available because the primary aims of the cohort (JPHC Study) did not include provision of evidence for suicide. In particular, the lack of any data on psychiatric disorders such as depression and alcohol misuse/dependence is the most important limitation. Second, because the numbers of suicides are small in each alcohol consumption group, the results might be overinterpreted. Third, ICD-10 suicide statistics might not be reliable because under-reporting and misclassification of suicide can occur (such as accidental drowning and poisonings) and any event in the undetermined intent category can

\section{CLINICAL IMPLICATIONS}

- There is a $U$-shaped association between alcohol consumption and subsequent suicide in middle-aged Japanese males.

- Non-drinkers are also candidates for suicide prevention efforts.

- There is no clearly different effect of alcohol consumption on suicide among non-flushers (inactive aldehyde dehydrogenase 2 ) and flushers (active aldehyde dehydrogenase 2).

\section{LIMITATIONS}

Potential confounding factors, especially psychiatric disorders and individual characteristics, may remain unaccounted.

- Suicide statistics may not be reliable because under-reporting and misclassification of suicide can occur.

- Since two metropolitan areas were excluded and the participants were middleaged Japanese men, our findings may not be generalisable to the entire Japanese urban population and to different generations and/or women.

TATSUO AKECHI, MD, PhD, Department of Psychiatry, Nagoya City University Medical School, Nagoya and Psycho-Oncology Division, National Cancer Centre Research Institute East, Chiba; MOTOKI IWASAKI, MD, PhD, Epidemiology and Prevention Division, Research Centre for Cancer Prevention and Screening, National Cancer Centre, Tokyo; YOSUKE UCHITOMI, MD, PhD, Psycho-Oncology Division, National Cancer Centre Research Institute East, Chiba; SHOICHIROTSUGANE, MD, PhD, Epidemiology and Prevention Division, Research Centre for Cancer Prevention and Screening, National Cancer Centre, Tokyo, Japan

Correspondence: Dr ShoichiroTsugane, Epidemiology and Prevention Division, Research Centre for Cancer Prevention and Screening, National Cancer Centre, 5-I-I Tsukiji, Chuo-ku, Tokyo I04-0045, Japan. Tel: +8I 33542 25II extension 3385; Fax: +8I 335478578 ; e-mail: stsugane@ncc.go.jp

(First received 12 October 2004, final revision 26 May 2005, accepted 8 June 2005)

be misclassified as suicide (Rockett \& Smith, 1993). However, this type of misclassification would be unlikely to have influenced the results because we observed similar results after taking deaths from undetermined causes into account. Fourth, there may be measurement errors in the alcohol consumption and other lifestyle factors resulting from the use of the questionnaire. In addition, the influence of modification of drinking habits during the follow-up period cannot be ignored. A reduction or increase of alcohol consumption might lead to attenuation of the true association. Fifth, since two of the metropolitan areas were excluded because of different definitions of the study population, our findings may not be directly generalisable to the entire Japanese urban population. However, our findings can at least be generalised to middle-aged and elderly Japanese men in rural areas.
Finally, because the participants were middle-aged Japanese men, the findings may not be applicable to those from different generations and/or to women.

\section{Further research}

Although there are several limitations, the observed association between alcohol consumption and suicide is still important, and future studies, particularly of the cooccurrence of alcohol dependence and mood disorders, should yield interesting results.

\section{ACKNOWLEDGEMENTS}

We thank all staff members in each study area and central offices for their cooperation and technical assistance. We also thank Dr S. Yamamoto of the Statistics and Cancer Control Division, Research Centre for Cancer Prevention and Screening, National Cancer Centre, Tokyo, Japan for his 
statistical advice, and Dr T. Hayashi of the Cellular Pathobiology Unit, Cellular Neurobiology Research Branch, Intramural Research Programme, National Institute on Drug Abuse, National Institute for Health and Dr T. Otani of the Epidemiology and Prevention Division, Research Centre for Cancer Prevention and Screening, National Cancer Centre, Tokyo, Japan for their helpful comments.

This study was supported by a Grant-in-Aid from the Cancer Research and Second-Term Comprehensive Ten-Year Strategy for Cancer Control from the Ministry of Labour, Health and Welfare of Japan.

Members of the JPHC Study Group (principal investigator S. Tsugane): M. Inoue, T. Hanaoka, S. Tsugane, S. Yamamoto and T. Sobue, Research Centre for Cancer Prevention and Screening, National Cancer Centre, Tokyo; J. Ogata, S. Baba, T. Mannami and A. Okayama, National Cardiovascular Centre, Suita; K. Miyakawa, F. Saito, A. Koizumi, Y. Sano and I. Hashimoto, Iwate Prefectural Ninohe Public Health Centre, Ninohe; Y. Miyajima, N. Suzuki, S. Nagasawa and Y. Furusugi, Akita Prefectural Yokote Public Health Centre, Yokote; H. Sanada, Y. Hatayama, F. Kobayashi, H. Uchino, Y. Shirai, T. Kondo, R. Sasaki and Y. Watanabe, Nagano Prefectural Saku Public Health Centre, Saku; Y. Kishimoto, E. Takara, T. Fukuyama, M. Kinjo and M. Irei, Okinawa Prefectural Ishikawa (Chubu) Public Health Centre, Ishikawa; K. Imoto, H. Yazawa, T. Seo, A. Seiko and F. Ito, Katsushika Public Health Centre, Tokyo; A. Murata, K. Minato, K. Motegi and T. Fujieda, Ibaraki Prefectural Kasama (Mito) Public Health Centre, Mito; K. Matsui, T. Abe and M. Katagiri, Niigata Prefectural Kashiwazaki Public Health Centre Kashiwazaki; M. Doi, A. Terao and Y. Ishikawa; Kochi Prefectural Tosayamada (Chuo-higashi) Public Health Centre, Tosayamada; H. Sueta, H. Doi, M. Urata, N. Okamoto and F. Ide, Nagasaki Prefectura Arikawa (Kamigoto) Public Health Centre, Arikawa; H. Sakiyama, N. Onga and H. Takaesu, Okinawa Prefectural Miyako Public Health Centre, Hirara; F. Horii, I. Asano, H. Yamaguchi, K. Aoki, S. Maruyama and M. Ichii, Osaka Prefectural Suita Public Health Centre, Suita; S. Matsushima and S. Natsukawa, Saku General Hospital, Usuda; S. Watanabe and M. Akabane, Tokyo University of Agriculture, Tokyo; M. Konishi and K. Okada, Ehime University, Matsuyama; $H$. Iso and $Y$. Honda, Tsukuba University, Tsukuba; H. Sugimura, Hamamatsu University, Hamamatsu; Y. Tsubono, Tohoku University, Sendai; M. Kabuto, National Institute for Environmental Studies, Tsukuba; S. Tominaga, Aichi Cancer Centre Research Institute, Nagoya; M. lida and W. Ajiki, Osaka Medical Centre for Cancer and Cardiovascular Disease, Osaka; S. Sato, Osaka Medical Centre for Health Science and Promotion, Osaka; N. Yasuda, Kochi University, Kochi; S. Kono, Kyushu University, Fukuoka; K. Suzuki, Research
Institute for Brain and Blood Vessels, Akita; Y. Takashima, Kyorin University, Mitaka; E. Maruyama, Kobe University, Kobe; the late M. Yamaguchi, Y. Matsumura and S. Sasaki, National Institute of Health and Nutrition, Tokyo; T. Kadowaki, Tokyo University, Tokyo, Japan.

\section{REFERENCES}

Andreasson, S., Romelsjo, A. \& Allebeck, P. (1991) Alcohol, social factors and mortality among young men British journal of Addiction, 86, 877-887.

Becker, B. J. (1994) Combining significance levels. In The Handbook of Research Synthesis (eds H. Cooper \& L. V. Hedges), pp. 215-230. New York: Russell Sage Foundation.

de Lorimier, A. A. (2000) Alcohol, wine and health American Journal of Surgery, 180, 357-361.

Ferguson, R. A. \& Goldberg, D. M. (1997) Genetic markers of alcohol abuse. Clinica Chimica Acta, 257 199-250.

Harris, E. C. \& Barraclough, B. (1997) Suicide as an outcome for mental disorders. A meta-analysis. British Journal of Psychiatry, 170, 205-228.

Hasegawa, Y., Higuchi, S., Matsushita, S., et al

(2002) Association of a polymorphism of the serotonin $\mathrm{IB}$ receptor gene and alcohol dependence with inactive aldehyde dehydrogenase-2. Journal of Neural

Transmission, 109, 513-521.

Heikkinen, M., Aro, H. \& Lonnqvist, J. (1993) Life events and social support in suicide. Suicide and Life Threatening Behaviour, 23, 343-358.

Higuchi, S., Parrish, K. M., Dufour, M. C., et al (1992) The relationship between three subtypes of the flushing response and DSM-III alcohol abuse in Japanese. Journal of Studies on Alcohol, 53, 553-560.

Huang, Y. Y., Oquendo, M. A., Friedman, J. M., et al (2003) Substance abuse disorder and major depression are associated with the human 5 - $\mathrm{HTIB}$ receptor gene (HTRIB) G86IC polymorphism.

Neuropsychopharmacology, 28, 163-169.

Hufford, M. R. (2001) Alcohol and suicidal behavior Clinical Psychology Reviews, 21, 797-8II.

Inskip, H. M., Harris, E. C. \& Barraclough, B.

(1998) Lifetime risk of suicide for affective disorder, alcoholism and schizophrenia. British Journal of Psychiatry, I72, 35-37.

Klatsky, A. L. \& Armstrong, M. A. (1993) Alcohol use, other traits, and risk of unnatural death: a prospective study. Alcoholism, Clinical and Experimental Research, 17, II56-1162.

Mann, J. J. (2002) A current perspective of suicide and attempted suicide. Annals of Internal Medicine, 136 302-3II.

Paffenbarger, R. S., Jr, King, S. H. \& Wing, A. L. (1969) Chronic disease in former college students. IX Characteristics in youth that predispose to suicide and accidental death in later life. American journal of Public Health and the Nation's Health, 59, 900-908.

Petitti, D. B. (1994) Statistical methods in metaanalysis. In Meta-Analysis, Decision Analysis, and CostEffectiveness Analysis: Methods for Quantitative Synthesis in Medicine, pp. 90-114. New York: Oxford University Press.

Rockett, I. R. \& Smith, G. S. (1993) Covert suicide among elderly Japanese females: questioning unintentional drownings. Social Science and Medicine, 36 |467-1472.

Ross, R. K., Bernstein, L., Trend, L., et al (1990) A prospective study of risk factors for traumatic deaths in a retirement community. Preventive Medicine, 19, 323-334.

Sato, S., Shouji, M., Senoh, E., et al (1990) A study of drinking habits of rural middle and old aged residents in Ibaraki prefecture. Relationship between problem drinking, depression and personality (part I) on male subjects. Japanese Journal of Alcohol and Drug Dependence, 25, 48-58.

Sobue, T., Yamamoto, S. \& Watanabe, S. (200I) Smoking and drinking habits among the JPHC study participants at baseline survey. Journal of Epidemiology, II (suppl. 6), S44-S56.

Takahashi, Y. (2002) Epidemiology of suicide. Clinical Neuroscience, 20, 544-546.

Takeshita, T., Morimoto, K., Mao, X. Q., et al (1993) Phenotypic differences in low $\mathrm{Km}$ aldehyde dehydrogenase in Japanese workers. Lancet, 34I, 837-838.

Tsubono, Y., Kobayashi, M., Sasaki, S., et al (2003) Validity and reproducibility of a self-administered food frequency questionnaire used in the baseline survey of the JPHC Study Cohort I. Journal of Epidemiology, 13 (suppl. I), SI25-SI33.

Tsugane, S., Fahey, M. T., Sasaki, S., et al (1999) Alcohol consumption and all-cause and cancer mortality among middle-aged Japanese men: seven-year followup of the JPHC study Cohort I. American Journal of Epidemiology, 150, I20I-1207.

Turvey, C. L., Conwell, Y., Jones, M. P., et al (2002) Risk factors for late-life suicide: a prospective, community-based study. American Journal of Geriatric Psychiatry, 10, 398-406.

Watanabe, S., Tsugane, S., Sobue, T., et al (200I) Study design and organization of the JPHC study. Journal of Epidemiology, II, S3-7.

World Health Organization (1992) Internationa Statistical Classification of Diseases and Related Health Problems (ICD-10). Geneva: WHO.

Yokoyama, A., Muramatsu, T., Ohmori, T., et al (1997) Reliability of a flushing questionnaire and the ethanol patch test in screening for inactive aldehyde dehydrogenase- 2 and alcohol-related cancer risk. Cancer Epidemiology, Biomarkers and Prevention, 6, II05-1107. 